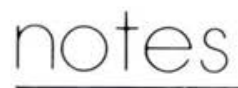

\title{
Evaluation de la teneur en uranium naturel des urines et des selles chez des sujets non exposés professionnellement
}

\author{
F. MICHAUD, M. ARCHIMBAUD, J. CHALABREYSSE* \\ (Manuscrit reçu le 4 février 1985)
}

\begin{abstract}
RÉSUME
Dans cette étude on a déterminé les taux d'uranium dans les urines et les selles d'une population non exposée professionnellement.

Deux populations habitant l'une sur un terrain sédimentaire, l'autre sur un terrain granitique ont été retenues.

On constate que les teneurs en uranium sont similaires pour les deux populations. Pour les urines de 24 heures, la teneur moyenne est de 0,1 à $0,2 \mu \mathrm{g} / \mathrm{g}$ de créatinine ; pour les selles, la quantité moyenne rejetée en 24 heures est comprise entre 1 et 3 ppm.
\end{abstract}

\section{ABSTRACT}

Uranium levels in excreta were determined in two non-exposed populations, living respectively on a sedimentary or a granitic ground. Uranium levels were similar in both populations. Mean levels in 24 hours'urines were $0.1-0.2 \mu \mathrm{g} / \mathrm{g}$ creatinin ; in feces, the mean amounts excreted in $24 \mathrm{~h}$ ranged between 1 and 3 ppm.

\section{BUT DU TRAVAIL}

De nombreux contrôles du personnel et de l'environnement sont pratiqués dans l'industrie mettant en œuvre des composés d'uranium. On mesure les taux d'uranium dans les urines et les selles pour évaluer le niveau de contamination interne. Les valeurs mesurées sont parfois très faibles lorsque les composés manipulés s'éliminent lentement (composés $W$ et $Y$ de la CIPR). II devient alors indispensable de connaître les teneurs normales de ces principaux excreta pour les sujets non exposés professionnellement, afin d'évaluer la part qui revient effectivement à l'exposition professionnelle.

* Commissariat à l'énergie atomique, Institut de protection et de sûreté nucléaire, Département de protection sanitaire, Service d'hygiène industrielle, BP 38,26701 Pierrelatte Cedex. 


\section{RESULTATS}

Nous avons choisi deux populations habitant à plus de $40 \mathrm{~km}$ d'installations nucléaires ou de mines d'uranium. Elles résident sur deux types de terrain :

- sédimentaire (région de Montpellier dans l'Hérault) ;

- granitique (région de St-Agrève dans l'Ardèche).

Nous avons analysé la teneur en uranium et le taux de créatinine dans les urines, l'uranium dans les selles et dans les eaux de consommation.

Les tableaux I et II indiquent les valeurs moyennes obtenues pour une dizaine de personnes dans chaque région granitique ou sédimentaire.

\section{CONCLUSION}

Cette étude a permis d'estimer les teneurs en uranium d'urines et de selles d'échantillons de populations de régions géologiques distinctes et exemptes d'industries nucléaires.

Les tableaux I et II rassemblent les résultats obtenus sur deux échantillons d'une dizaine de personnes pour chaque région. Compte tenu des faibles écarts observés pour les moyennes et de l'importance des écarts types, nous conviendrons que les teneurs en uranium sont similaires pour les deux populations.

Pour les urines de 24 heures, la teneur moyenne est de 0,1 à $0,2 \mu \mathrm{g} / \mathrm{g}$ de créatinine; pour les selles, la quantité moyenne rejetée en 24 heures est comprise entre 1 et $3 \mathrm{ppm}$.

TABLEAU I

Moyenne maximale et écart type des teneurs en uranium en région granitique

\begin{tabular}{|c|c|c|}
\hline & Urines & Selles de $24 \mathrm{~h}$ \\
\hline $\begin{array}{l}\text { Moyenne des } \\
\text { quantités d'uranium }\end{array}$ & $0,2 \mu \mathrm{g} / \mathrm{g}$ de créatinine & $\begin{array}{l}0,4 \mu \mathrm{g} / \mathrm{g} \text { de cendres } \\
1,2 \mu \mathrm{g} \text { en totalité }\end{array}$ \\
\hline $\begin{array}{l}\text { Maximum des } \\
\text { quantités d'uranium }\end{array}$ & $0,4 \mu \mathrm{g} / \mathrm{g}$ de créatinine & $\begin{array}{l}0,7 \mu \mathrm{g} / \mathrm{g} \text { de cendres } \\
3,1 \mu \mathrm{g} \text { en totalité }\end{array}$ \\
\hline Ecart type & $0,09 \mu \mathrm{g} / \mathrm{g}$ de créatinine & $\begin{array}{l}0,15 \mu \mathrm{g} / \mathrm{g} \text { de cendres } \\
0,9 \mu \mathrm{g} \text { en totalité }\end{array}$ \\
\hline
\end{tabular}

TABLEAU ॥

Moyenne maximale et écart type des teneurs en uranium en région sédimentaire

\begin{tabular}{|c|c|c|}
\hline \multirow{2}{*}{$\begin{array}{c}\text { Moyenne des } \\
\text { quantités d'uranium }\end{array}$} & Urines & \multicolumn{1}{c|}{ Selles de $24 \mathrm{~h}$} \\
\cline { 2 - 3 } $\begin{array}{c}\text { Maximum des } \\
\text { quantités d'uranium }\end{array}$ & $0,1 \mu \mathrm{g} / \mathrm{g}$ de créatinine & $\begin{array}{l}0,5 \mu \mathrm{g} / \mathrm{g} \text { de cendres } \\
2,5 \mu \mathrm{g} \text { en totalité }\end{array}$ \\
Ecart type & $0,2 \mu \mathrm{g} / \mathrm{g}$ de créatinine & $\begin{array}{l}0,8 \mathrm{~g} / \mathrm{g} \text { de cendres } \\
6,6 \mu \mathrm{g} \text { en totalité } \\
0,2 \mu \mathrm{g} / \mathrm{g} \text { de cendres } \\
1,9 \mu \mathrm{g} \text { en totalité }\end{array}$ \\
\hline
\end{tabular}

